\title{
Social stress exacerbates the aversion to painful experiences in rats exposed to chronic pain: The role of the locus coeruleus
}

\author{
Lidia Bravo $^{\mathrm{a}, \mathrm{b}}$, Cristina Alba-Delgado ${ }^{\mathrm{a}, \mathrm{b}}$, Sonia Torres-Sanchez ${ }^{\mathrm{a}, \mathrm{b}}$, Juan Antonio Mico ${ }^{\mathrm{a}, \mathrm{b}}$, Fani L. Neto ${ }^{\mathrm{c}, \mathrm{d}}$, \\ Esther Berrocoso ${ }^{\mathrm{a}, \mathrm{b}, *}$ \\ a Neuropsychopharmacology and Psychobiology Research Group, University of Cadiz, Cadiz, Spain

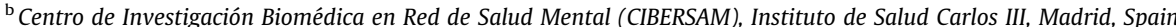 \\ ${ }^{\mathrm{c}}$ Departamento de Biologia Experimental, Centro de Investigação Médica-Faculdade de Medicina (CIM-FMUP), Universidade do Porto, Porto, Portugal \\ ${ }^{\mathrm{d}}$ Morphysiology of the Somatosensory System Group, Instituto de Biologia Molecular e Celular (IBMC), Universidade do Porto, Porto, Portugal
}

Sponsorships or competing interests that may be relevant to content are disclosed at the end of this article.

\section{A R T I C L E I N F O}

\section{Article history:}

Received 31 January 2013

Received in revised form 28 May 2013

Accepted 11 June 2013

\section{Keywords:}

Neuropathic pain

Stress

Locus coeruleus

Affective pain

Tyrosine hydroxylase

Gephyrin

\begin{abstract}
A B S T R A C T
Stressful experiences seem to negatively influence pain perception through as yet unknown mechanisms. As the noradrenergic locus coeruleus (LC) nucleus coordinates many components of the stress response, as well as nociceptive transmission, we evaluated whether the sensory and affective dimension of chronic neuropathic pain worsens in situations of stress due to adaptive changes of LC neurons. Accordingly, male rats were socially isolated for 5 weeks, and in the last 2 weeks, neuropathic pain was induced by chronic constriction injury. In this situation of stress, chronic pain selectively heightened the animal's aversion to painful experiences (affective pain), as measured in the place escape/avoidance test, although no changes were observed in the sensory dimension of pain. In addition, electrophysiological recordings of LC neurons showed a low tonic but exacerbated nociceptive-evoked activity when the injured paw was stimulated. These changes were accompanied by an increase in tyrosine hydroxylase and gephyrin expression in the LC. Furthermore, intra-LC administration of bicuculline, a $\gamma$-aminobutyric acid-A receptor antagonist, attenuated the negative affective effects of pain. These data show that changes in the LC are greater than those expected from the simple summation of each independent factor (pain and stress), revealing mechanisms through which stressors may exacerbate pain perception without affecting the sensorial dimension.
\end{abstract}

(C) 2013 International Association for the Study of Pain. Published by Elsevier B.V. All rights reserved.

\section{Introduction}

Chronic neuropathic pain is a common problem for which adequate treatments remain elusive. This is because the relationship between the nociceptive input that originates at the injured nerve and the resulting subjective pain experience is far from linear. Stress has consistently been shown to be a factor that contributes to the maintenance and amplification of the severity of pain $[17,39]$. However, most such reports are correlational and the underlying biological mechanisms remain unclear.

The main source of noradrenaline in the central nervous system is the pontine locus coeruleus nucleus (LC), a structure that is closely linked to pain, stress, and emotions. Indeed, ascending nociceptive inputs from the LC reach the forebrain, while its descending projections innervate the spinal cord [33]. In addition,

* Corresponding author. Address: Department of Psychology, Psychobiology Area Campus Universitario Río San Pedro s/n, 11510 Puerto Real (Cadiz), Spain. Tel.: +34 956015224; fax: +34 956015225 .

E-mail address: esther.berrocoso@uca.es (E. Berrocoso). it is a structure that is essential to the cognitive branch of the stress response and it is thought to be dysregulated in mental disorders such as depression, anxiety, and posttraumatic stress disorder $[24,40]$. In this sense, we have recently shown that long-term neuropathic pain leads to anxio-depressive-like behaviour that is temporally accompanied by an impairment of the LC [2]. Moreover, antidepressants that inhibit the reuptake of noradrenaline can normalize the nociceptive-evoked responses of LC neurons in rats that experience neuropathic pain [3]. Thus, we hypothesized that stress may trigger functional and/or structural changes in the LC that amplify pain. Accordingly, in this study we have evaluated the effect of stress (social isolation) on the pain response and on the electrophysiological/neurochemical properties of LC in neuropathic rats.

\section{Methods}

\subsection{Animals and experimental design}

Male Sprague-Dawley rats (University of Cadiz [ES110120000210], Spain) were used in these studies, in accordance with the 
guidelines of the European Commission's directive (2010/63/EU) and the Spanish Law (RD 1201/2005). All the experimental protocols were approved by the Committee for Animal Experimentation at the University of Cadiz (Spain). Animals (weighing 200-220 g at the beginning of the experiment) were housed under standard laboratory conditions (water and food ad libitum, a constant room temperature of $22 \pm 1^{\circ} \mathrm{C}$, and a 12-hour light/dark cycle). Neuropathic pain was induced by chronic constriction injury (CCI $[5,6])$ whereby the common left sciatic nerve was ligated. In sham, an identical dissection was performed in the left paw, but the sciatic nerve was not ligated. Stressed rats were housed individually in standard plastic cages $(22 \times 22 \times 14.5 \mathrm{~cm})$, while control animals were housed in groups of 4 in similar cages $(21.5 \times$ $46.5 \times 14.5 \mathrm{~cm})$. Control rats were housed according to their pain condition, that is, animals of the same cage belonged to the same group (sham or CCI). The stress regime lasted for 5 weeks and, where appropriate, neuropathic pain was induced during the third week of stress (Fig. 1A). Thus, 4 groups were evaluated: Sham-control, Sham-stress, CCI-control, and CCI-stress.

\subsection{Nociceptive behavioural testing}

Sensory pain was evaluated before, as well as 1 and 2 weeks after nerve injury (weeks 3,4 , and 5 of stress regime). The presence of mechanical allodynia was measured using an automatic apparatus to apply the von Frey test (Dynamic Plantar Aesthesiometer, Ugo Basile, Italy). This device uses a single nonflexible filament that applies increasing force (from 0 to $50 \mathrm{~g}$ ) against the plantar surface of the hind paw over a 20 -second period. The nocifensive paw withdrawal response automatically turns off the stimulus, and the mechanical pressure that evoked the response is recorded [8]. Two measurements were taken for each paw at 5-minute intervals and the mean value was considered the nociceptive threshold. Cold allodynia was evaluated applying a drop of acetone $(100 \mu \mathrm{L})$ with a pipette to the centre of the ventral surface of the hind paw [16]. Acetone was applied alternately 5 times to each hind paw, at 5-minute intervals, and the responses were recorded over a 1-minute period according to the following scale: 0 , no response; 1 , quick withdrawal, flick, or stamp of the paw; 2, prolonged

A

\begin{tabular}{|c|c|c|c|c|c|}
\hline Weeks & 1 & 2 & 3 & 4 & 5 \\
\hline \multirow{5}{*}{ Stress } \\
\cline { 2 - 5 }
\end{tabular}

B

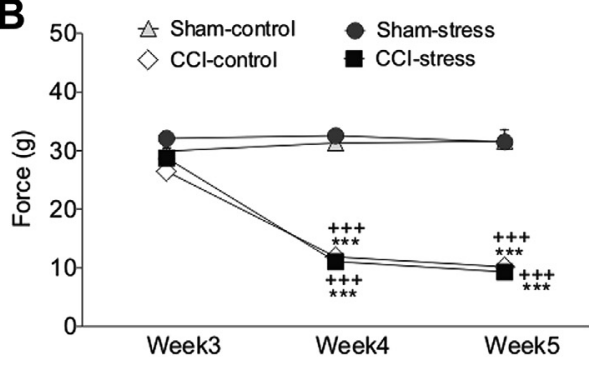

D

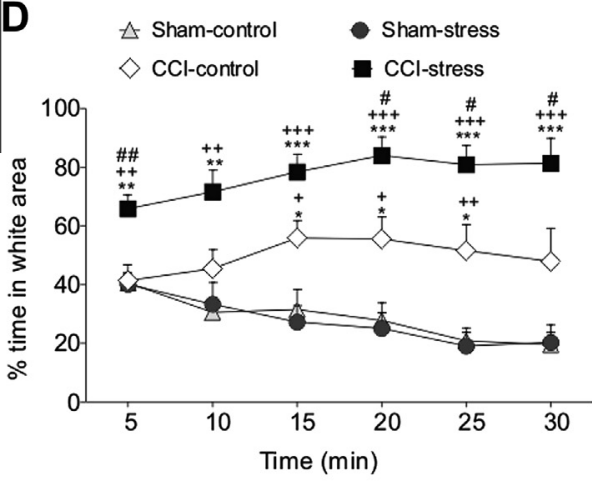

$\mathbf{F}$

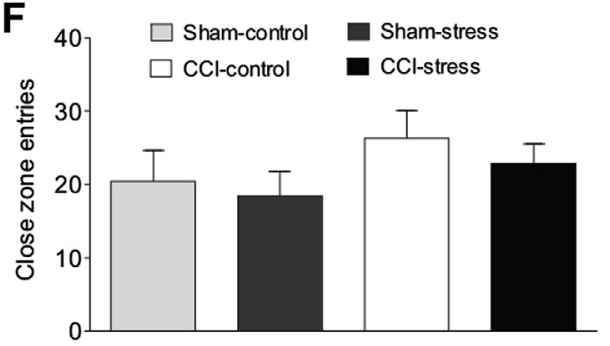

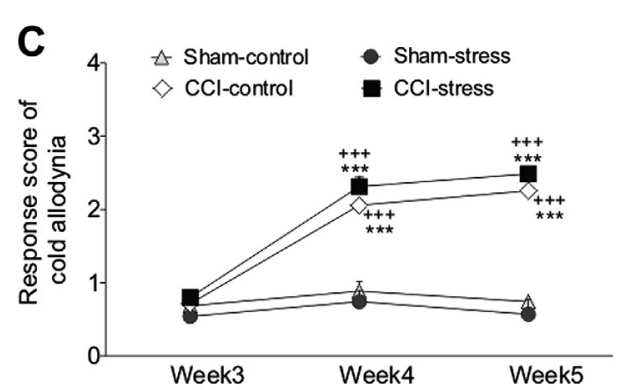

E
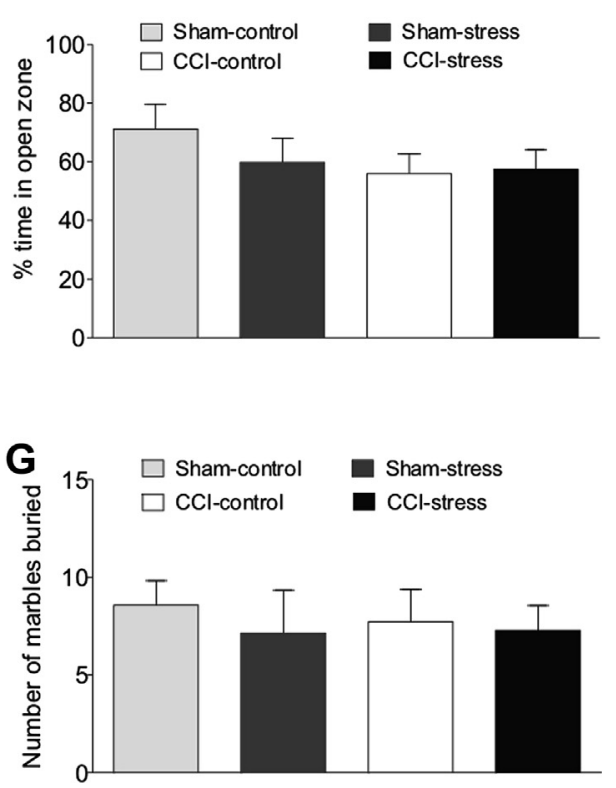

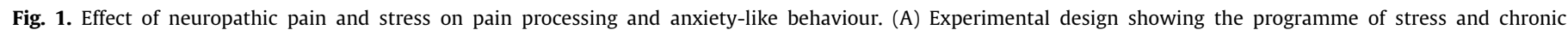

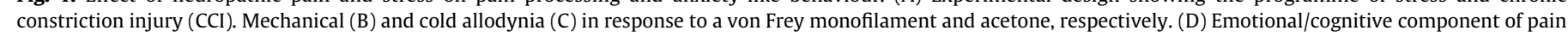

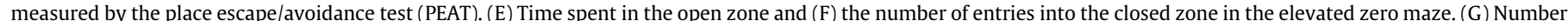

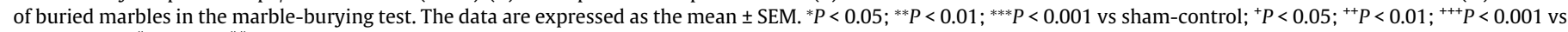
sham-stress; ${ }^{\#} P<0.05$; ${ }^{\# \#} P<0.01$, vs CCI-control ( $\mathrm{n}=7-8$ per group). 
withdrawal or repeated flicking of the paw; 3 , repeated flicking of the paw with persistent licking directed at the ventral side of the paw. The cumulative score for each rat was obtained by summing the scores and dividing them by the number of assays.

\subsection{Emotional behavioural testing}

One set of animals was tested 5 weeks after stress in the place escape/avoidance test (PEAT) to explore the affective-cognitive dimension of pain $[2,23]$. Animals were placed in the centre of a $60 \times 30 \times 30 \mathrm{~cm}$ Plexiglas chamber on an elevated metal grid. One half of the chamber was white and the other half of the chamber was black. The rats were allowed to move freely throughout the chamber over the 30-minute test period, during which they were mechanically stimulated at 15 -second intervals with a von Frey monofilament (60 g) on the plantar surface of the hind paw, depending on which area they were in. Thus, if the animals were in the black side of the chamber, the left (ipsilateral) paw was stimulated, whereas the right (contralateral) paw was stimulated when they were in the white side. Each 30-minute test session was recorded and subsequently analyzed with SMART (Spontaneous Motor Activity Recording and Tracking) software. We scored the proportion of time spent in the white area, binned into 5-minute time intervals.

To evaluate anxiety-like behaviour, the elevated zero maze test was performed after 5 weeks of stress [2]. After the animals were placed in a closed section, they were allowed to investigate the maze for 5 minutes, during which the time spent in the open zone was evaluated. Additionally, the number of close zone entries was recorded as indicative of motor behaviour. In addition, a marbleburying test was carried out [27] in which the rats were placed individually in a cage $(21.5 \times 46.5 \times 14.5 \mathrm{~cm})$ that contained 5 $\mathrm{cm}$ deep bedding and 20 marbles $(1.5 \mathrm{~cm}$ in diameter), arranged in 5 by 4 rows. The test was performed over 30 minutes under white light, after which the rats were removed and the buried marbles counted. Marbles were considered buried if they were at least three-quarters covered with bedding. A decrease in the proportion of time spent in the open arms or an increase in the numbers of marbles buried was considered as an index of anxiety behaviour in the elevated zero maze test and marble buried test, respectively.

\subsection{Intra-LC drug administration}

At 4 days after $\mathrm{CCI}$ surgery, a parallel group of rats were anaesthetized and placed in a stereotaxic frame in order to implant a stainless steel guide cannula unilaterally into the right (contralateral) LC (relative to lambda and dural surface: anteroposterior $-3.7 \mathrm{~mm}$, midline $-1.1 \mathrm{~mm}$, and dorsoventral $-6.2 \mathrm{~mm}$; [32]). The contralateral LC was pharmacologically explored because most nociceptive inputs from periphery afferents decussate in the dorsal horn at spinal cord level and then; nociceptive information ascends to the contralateral LC neurons [33]. The cannula was fixed to the skull with polyacrylic cement, anchored with stainless steel screws, and it remained closed due to the insertion of a stainless steel wire. A dose of the $\gamma$-aminobutyric acid (GABA)-A receptor antagonist, 1(S), 9(R)-(-)-bicuculline methiodide (10 ng per $0.5 \mu \mathrm{L}$; Sigma-Aldrich Chemicals, St. Louis, MO, USA), was dissolved in artificial cerebrospinal fluid and administered to the LC 5 minutes before the PEAT was performed. In addition, mechanical allodynia was evaluated using the von Frey test before and after the PEAT. To administer the drugs, animals were held gently while the wire was removed and a removable injector was inserted into the guide cannula, extending $1 \mathrm{~mm}$ beyond the guide tip. The injector was linked to a $10-\mu \mathrm{L}$ Hamilton syringe and the drug solution was injected over 30 seconds. The injector remained in the guide cannula for a further 1 minute after infusion to allow the compound to spread. At the end of each experiment, $0.5 \mu \mathrm{L}$ of Pontamine Sky Blue was used to mark the cannula site. The location of the injection site was verified as being within the LC for all of the animals that were included in this study.

\subsection{Electrophysiological $L C$ recordings}

Single-unit extracellular recordings of LC neurons contralateral to the operated hind paw were obtained 5 weeks after the start of the stress regime in a parallel set of animals [1,7]. The recording electrode was lowered into the LC (anteroposterior: $-3.7 \mathrm{~mm}$, mediolateral: $-1.1 \mathrm{~mm}$ relative to lambda and $5.0-7.0 \mathrm{~mm}$ from brain surface after dura removal) [32]. LC neurons were identified on the basis of well-established criteria [12]: long-duration action potential $(>2 \mathrm{~ms})$, spontaneous firing at a regular rhythm, a slow firing rate between 0.5 and $5 \mathrm{~Hz}$, and characteristic spikes with a longlasting positive-negative waveform (Fig. 2B). Firing patterns were analyzed offline using the computer software Spike2 (Cambridge Electronic Design, Cambridge, UK). At the end of each experiment, the location of the recording site was verified (Fig. 2A). Only measurements from cells within the LC were included in this study.

When a single unit was isolated, the tonic and the phasic activity of LC neurons were measured. The coefficient of variation (\%) was expressed as the ratio of the SD to the mean interval value of an interspike time-interval histogram; the burst firing discharge was defined according to Grace and Bunney (1984) [20]; and histograms of mean interspike interval (ISI) were defined as the frequency of spike intervals for consecutive spikes. Phasic activation (burst of action potentials) was elicited by paw compression (ipsilateral or contralateral to injured paw) for 1 second between the ends of a pair of 15-cm surgical forceps (Ref. 501742-G; World Precision Instruments, Hertfordshire, UK) by applying pressure midway along the forceps such that the opposite sides of the forceps, at this midpoint, came into contact. This sensory-evoked discharge was followed by a postactivation inhibition or suppression period (Fig. 2C) [21,41].

In another set of rats, intravenous (i.v.) dose-response curves were constructed for UK14,304 ( $\alpha_{2}$-adrenoceptor agonist; 2.5 $80.0 \mu \mathrm{g} / \mathrm{kg}$; Tocris Bioscience, Bristol, UK) and desipramine (noradrenaline reuptake inhibitor; $0.05-1.6 \mathrm{mg} / \mathrm{kg}$; Sigma-Aldrich Chemicals) in doubling dose. When complete inhibition was achieved, LC neuron activity was reversed by i.v. administration of the $\alpha_{2}$-adrenoceptor antagonists, RX821002 and idazoxan, respectively (Sigma Chemical, St. Louis, Missouri). The effective dose for eliciting $50 \%\left(E_{50}\right)$ of $E_{\max }(100 \%)$ was analyzed for the best nonlinear fit to the logistic 3-parameter equation [31]. Only one dose-response curve was obtained per rat.

\subsection{Immunohistochemistry}

One set of animals was perfused at the end of the experiments [1] and 1 in each 4 free-floating sequential sections (30 $\mu \mathrm{m})$ were incubated with a sheep antityrosine hydroxylase (TH, 1:1000; Abcam, Cambridge, UK) or a mouse monoclonal antigephyrin (mAb7a, 1:400; Synaptic Systems, Goettingen, Germany). Subsequently, the sections were incubated with, respectively, a donkey antisheep Alexa Fluor 568 (1:1000; Invitrogen, Madrid, Spain), or with biotinylated donkey antimouse antibody (1:200; Jackson Immunoresearch Lab, West Grove, PA, USA) followed by Streptavidin 488 (1:1000; Invitrogen), before they were washed and coverslipped with fluorogel aqueous mounting medium. TH-immunoreactivity (TH-IR) was visualised on an Olympus BX60 microscope (Olympus America, Center Valley, PA, USA) equipped with a U-MNU filter system, and the number of cell bodies labelled (TH-IR) were counted manually in an average of 6 sections of LC per animal ( $n=4-5$ rats per group), by an experimenter blind to the conditions. The 

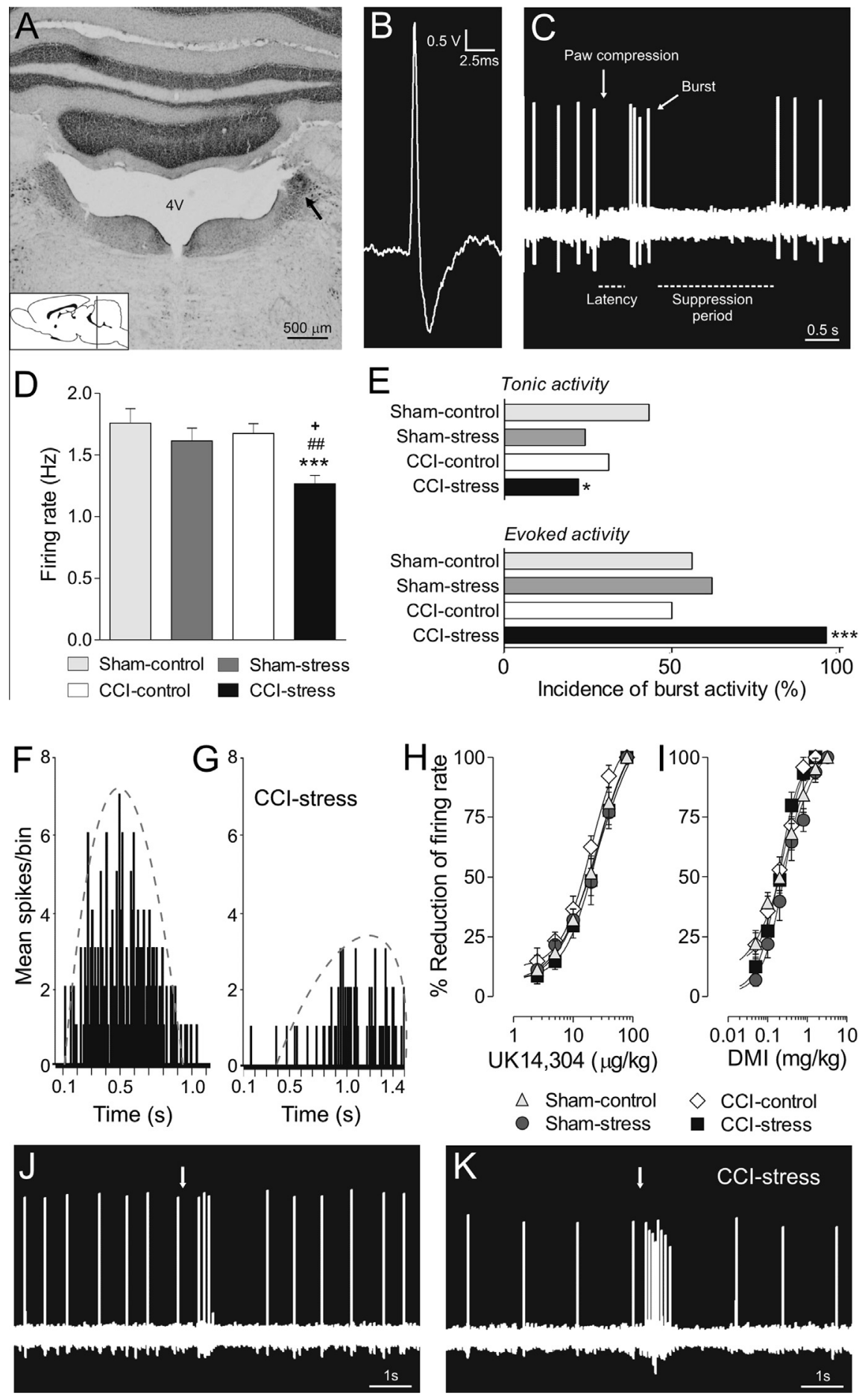

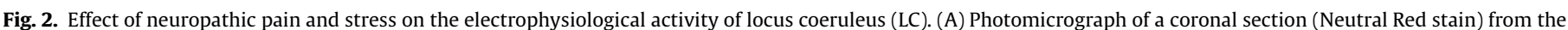

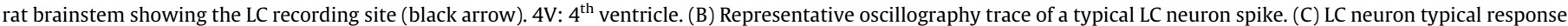

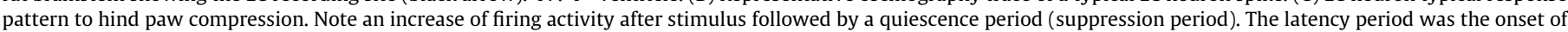

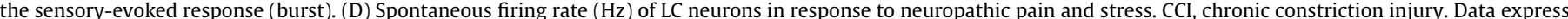

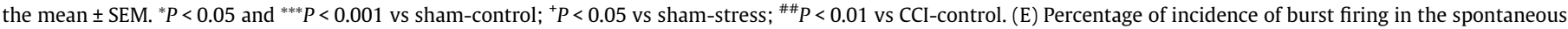

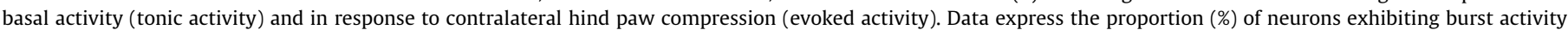

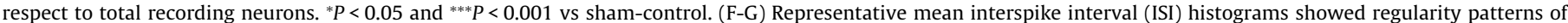

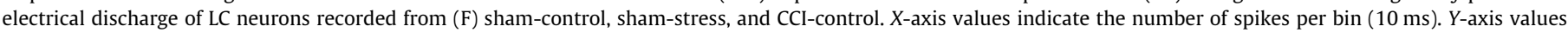

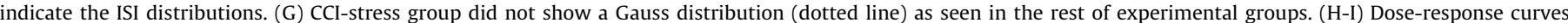

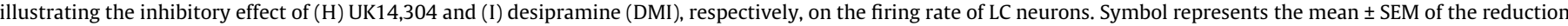

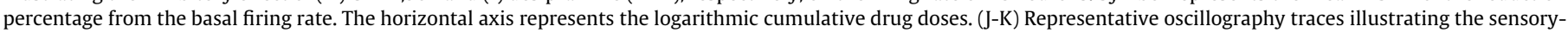

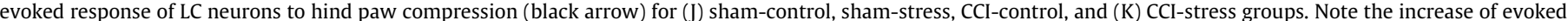
response in CCI-stress group; 12-16 animals per group were used for the electrophysiological recordings (see details in Table 2). 
Table 1

Analysis of variance (ANOVA) summary.

\begin{tabular}{|c|c|c|c|c|c|}
\hline \multirow[t]{2}{*}{ 2- , 3- or 4-way ANOVA } & \multirow[t]{2}{*}{ von Frey test } & \multirow[t]{2}{*}{ Acetone test } & \multirow[t]{2}{*}{ PEAT } & \multicolumn{2}{|c|}{ Bicuculline (10 ng/0.5 $\mu \mathrm{L}$ ) } \\
\hline & & & & von Frey test & PEAT \\
\hline $\mathrm{CCI}$ & $\mathbf{F}_{(1.24)}=335.12^{* * *}$ & $\mathbf{F}_{(1.24)}=204.57^{* * *}$ & $\mathbf{F}_{(1.26)}=47.89^{* * *}$ & $\mathrm{~F}_{(1.54)}=507.82^{* * *}$ & $\mathrm{~F}_{(1.54)}=28.30^{* * *}$ \\
\hline Stress & $F_{(1.24)}=0.64$ & $F_{(1.24)}=0.06$ & $F_{(1.26)}=6.70^{*}$ & $F_{(1.54)}=2.71$ & $F_{(1.54)}=4.93^{*}$ \\
\hline Treatment & $\sim$ & $\sim$ & $\sim$ & $F_{(1.54)}=0.00$ & $\mathrm{~F}_{(1.54)}=17.57^{* * *}$ \\
\hline $\mathrm{CCI} \times$ stress & $\mathrm{F}_{(1.24)}=0.30$ & $F_{(1.24)}=5.19^{*}$ & $F_{(1.26)}=7.69^{*}$ & $F_{(1.54)}=0.02$ & $F_{(1.54)}=3.33$ \\
\hline $\mathrm{CCI} \times$ treatment & $\sim$ & $\sim$ & $\sim$ & $F_{(1.54)}=0.02$ & $\mathrm{~F}_{(1.54)}=6.35^{*}$ \\
\hline Stress $\times$ treatment & $\sim$ & $\sim$ & $\sim$ & $\mathrm{F}_{(1.54)}=2.45$ & $F_{(1.54)}=0.05$ \\
\hline $\mathrm{CCI} \times$ stress $\times$ treatment & $\sim$ & $\sim$ & $\sim$ & $F_{(1.54)}=3.00$ & $F_{(1.54)}=1.62$ \\
\hline Time & $\mathbf{F}_{(2.48)}=57.02^{* * *}$ & $F_{(2.48)}=74.66^{* * *}$ & $F_{(5.130)}=1.40$ & $\mathrm{~F}_{(1.54)}=0.79$ & $\mathrm{~F}_{(5.270)}=3.28^{* *}$ \\
\hline Time $\times$ CCI & $F_{(2.48)}=66.83^{* * *}$ & $\mathbf{F}_{(2.48)}=56.64^{* * *}$ & $\mathbf{F}_{(5.130)}=8.97^{* * *}$ & $F_{(1.54)}=3.24$ & $\mathrm{~F}_{(5.270)}=3.51^{* *}$ \\
\hline Time $\times$ stress & $\mathrm{F}_{(2.48)}=1.25$ & $\mathrm{~F}_{(2.48)}=0.16$ & $F_{(5.130)}=0.37$ & $F_{(1.54)}=0.11$ & $\mathrm{~F}_{(5.270)}=0.97$ \\
\hline Time $\times$ treatment & $\sim$ & $\sim$ & $\sim$ & $F_{(1.54)}=1.36$ & $F_{(5.270)}=0.18$ \\
\hline Time $\times \mathrm{CCI} \times$ stress & $F_{(2.48)}=0.18$ & $F_{(2.48)}=0.20$ & $F_{(5.130)}=0.18$ & $\mathrm{~F}_{(1.54)}=0.60$ & $\mathrm{~F}_{(5.270)}=0.65$ \\
\hline Time $\times \mathrm{CCI} \times$ treatment & $\sim$ & $\sim$ & $\sim$ & $F_{(1.54)}=0.06$ & $\mathrm{~F}_{(5.270)}=3.11^{* *}$ \\
\hline Time $\times$ stress $\times$ treatment & $\sim$ & $\sim$ & $\sim$ & $F_{(1.54)}=1.86$ & $\mathrm{~F}_{(5.270)}=1.37$ \\
\hline Time $\times \mathrm{CCI} \times$ stress $\times$ treatment & $\sim$ & $\sim$ & $\sim$ & $F_{(1.54)}=1.26$ & $F_{(5.270)}=0.94$ \\
\hline Two-way ANOVA & $\mathrm{CCI}$ & Stress & $\mathrm{CCI} \times$ stress & & \\
\hline \multicolumn{6}{|l|}{ Behavioural tests } \\
\hline Elevated zero maze & $F_{(1.24)}=1.36$ & $F_{(1.24)}=0.43$ & $\mathrm{~F}_{(1.24)}=0.71$ & & \\
\hline Marble-burying test & $F_{(1.24)}=0.05$ & $\mathrm{~F}_{(1.24)}=0.32$ & $\mathrm{~F}_{(1.24)}=0.09$ & & \\
\hline \multicolumn{6}{|l|}{ Electrophysiological recordings } \\
\hline \multicolumn{6}{|l|}{ Tonic activity } \\
\hline Firing rate & $F_{(1.194)}=6.15^{*}$ & $F_{(1.194)}=9.81^{* *}$ & $F_{(1.194)}=2.48$ & & \\
\hline Variation coefficient & $\mathrm{F}_{(1.194)}=0.65$ & $F_{(1.194)}=4.00^{*}$ & $F_{(1.194)}=4.71^{*}$ & & \\
\hline Burst rate & $F_{(1.194)}=0.54$ & $F_{(1.194)}=11.74^{* * *}$ & $F_{(1.194)}=0.04$ & & \\
\hline Spikes per burst & $F_{(1.57)}=4.19 *$ & $F_{(1.57)}=0.04$ & $\mathrm{~F}_{(1.57)}=0.04$ & & \\
\hline$\%$ Spikes in burst & $\mathrm{F}_{(1.57)}=1.48$ & $\mathrm{~F}_{(1.57)}=1.93$ & $\mathrm{~F}_{(1.57)}=0.48$ & & \\
\hline \multicolumn{6}{|c|}{ Evoked activity after ipsilateral paw compression } \\
\hline Latency & $F_{(1.85)}=11.3^{*}$ & $F_{(1.85)}=0.46$ & $\mathrm{~F}_{(1.85)}=0.11$ & & \\
\hline Spikes/s per burst & $F_{(1.85)}=3.60$ & $\mathrm{~F}_{(1.85)}=2.80$ & $F_{(1.85)}=5.14^{*}$ & & \\
\hline Suppression period & $F_{(1.85)}=3.60$ & $\mathrm{~F}_{(1.85)}=1.90$ & $\mathrm{~F}_{(1.85)}=2.90$ & & \\
\hline \multicolumn{6}{|c|}{ Evoked activity after contralateral paw compression } \\
\hline Latency & $F_{(1.21)}=2.6$ & $\mathrm{~F}_{(1.21)}=0.1$ & $F_{(1.21)}=0.0$ & & \\
\hline Spikes/s per burst & $F_{(1.21)}=2.6$ & $F_{(1.21)}=0.1$ & $F_{(1.21)}=0.1$ & & \\
\hline Suppression period & $F_{(1.21)}=1.2$ & $F_{(1.21)}=0.0$ & $F_{(1.21)}=0.0$ & & \\
\hline \multicolumn{6}{|l|}{ Immunohistochemistry studies } \\
\hline $\mathrm{TH}$ & $F_{(1.13)}=6.62^{*}$ & $F_{(1.13)}=6.94^{*}$ & $F_{(1.13)}=16.05^{* *}$ & & \\
\hline Gephyrin & $F_{(1.12)}=16.46^{* *}$ & $\mathrm{~F}_{(1.12)}=2.76$ & $\mathrm{~F}_{(1.12)}=1.02$ & & \\
\hline
\end{tabular}

CCI, chronic constriction injury; TH, tyrosine hydroxylase; PEAT, place escape/avoidance test.

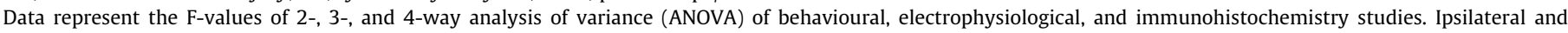
contralateral terms refer to the injured paw. Significant results are indicated in bold. ${ }^{*} P<0.05,{ }^{* *} P<0.01$, and ${ }^{* * *} P<0.001$

average number of TH-IR cells in each LC was used for statistical purposes. The expression of gephyrin was detected by using a Leica Spectra Confocal microscope (Leica Microsystems Inc, Buffalo Grove, IL, USA). A Z-stack consisting of 5-6 optical sections was acquired with a $63 \mathrm{X}$ oil immersion from 2 randomized LC per rat ( $n=2$ rats per group). Each $z$ plane was analyzed by using a Macro generated from Image software (National Institutes of Health, Bethesda, MD, USA), which converted the image to 8 bits and normalized the background in respect to the control. Quantification of gephyrin expression was performed as described elsewhere [35]. Thus, after normalizing the background, the number of clusters was quantified (minimal cluster size of $0.1 \mu \mathrm{m}^{2}$ ) by using a threshold ( 70 on a $0-255$ greyscale) for signal intensity that maximized the selection of puncta and minimized the background noise. The results were expressed as the number of clusters $/ 100 \mu \mathrm{m}^{2}$.

\subsection{Statistical analysis}

Data represent the mean \pm SEM, and all the results were analyzed using either 2-, 3-, or 4-way analysis of variance, with or without repeated measures, as appropriate. The factors of variance were: CCI (between-groups), stress (between-groups), treatment (bicuculline treatment, between-groups), and time (withingroups) (see Table 1 for detailed statistical analysis). All further post hoc analyses were carried out using a Bonferroni post hoc test.
The burst incidence was analyzed by Fisher's exact test. The level of significance considered was $P<0.05$.

\section{Results}

\subsection{Nociceptive and emotional behavioural response}

Animals subjected to 3 weeks of stress exhibited similar pain thresholds when compared with nonstressed animals (control vs stress groups: Fig. 1B-C). However, neuropathic rats (CCI-control and CCI-stress) exhibited pronounced mechanical allodynia of the operated hind paw in the von Frey test at week 4 and 5 of the stress regime $(P<0.001$; Fig. $1 \mathrm{~B})$. Moreover, they scored significantly higher than the sham-control or sham-stress in the acetone test $(P<0.001$; Fig. $1 \mathrm{C})$, reflecting cold allodynia. Overall, the sensorial nociceptive responses of the ipsilateral hind paw were similar in both CCI groups (CCI-control and CCI-stress), and no decrease in the pain threshold was observed in sham-stress. We evaluated the affective-cognitive dimension of pain using the PEAT at week 5 (2 weeks after neuropathy induction) (Fig. 1D). As expected, CCI-control animals demonstrated a greater tendency to escape from the dark side than the sham-operated rats. Interestingly, the CCI-stress rats spent even more time within the white area than the CCI-control animals, with significance at 5 , 20,25 , and 30 minutes $(P<0.01, P<0.05, P<0.05, P<0.05$, 
Table 2

Electrophysiological properties of locus coeruleus neurons.

\begin{tabular}{|c|c|c|c|c|}
\hline & Sham-control $\{16\}$ & CCI-control $\{12\}$ & Sham-stress $\{16\}$ & CCI-stress $\{16\}$ \\
\hline \multicolumn{5}{|l|}{ Tonic activity } \\
\hline Firing rate $(\mathrm{Hz})$ & $1.8 \pm 0.1$ & $1.7 \pm 0.1$ & $1.6 \pm 0.1$ & $1.2 \pm 0.1^{* * *+\# \#}$ \\
\hline Variation coefficient (\%) & $37.3 \pm 1.6$ & $39.4 \pm 1.4$ & $37.5 \pm 1.6$ & $33.0 \pm 1.5^{\#}$ \\
\hline \multicolumn{5}{|l|}{ Burst firing activity } \\
\hline Incidence (\%) & $42.3(22 / 52)$ & $30.7(16 / 52)$ & $28.6(12 / 42)$ & $21.2(11 / 52)^{*}$ \\
\hline Burst rate (burst/ms) & $11.0 \pm 2.0$ & $10.0 \pm 3.0$ & $5.0 \pm 1.0$ & $3.0 \pm 1.0 * \#$ \\
\hline Spikes per burst & $2.1 \pm 0.1$ & $2.0 \pm 0.0$ & $2.2 \pm 0.1$ & $2.0 \pm 0.0$ \\
\hline Spikes in burst (\%) & $2.1 \pm 0.3$ & $2.9 \pm 0.4$ & $1.8 \pm 0.4$ & $2.1 \pm 0.4$ \\
\hline \multicolumn{5}{|c|}{ Evoked activity after ipsilateral paw compression } \\
\hline Incidence $(\%)$ & $56.3(31 / 55)$ & $50.0(20 / 40)$ & $61.9(13 / 21)$ & $96.1(25 / 26)^{* * *}$ \\
\hline Latency (ms) & $581.9 \pm 64.9$ & $400.8 \pm 32.3$ & $561.1 \pm 76.5$ & $315.8 \pm 34.1^{* *+}$ \\
\hline Spikes/s per burst & $3.4 \pm 0.2$ & $4.3 \pm 0.3$ & $3.2 \pm 0.3$ & $4.6 \pm 0.4^{*+\#}$ \\
\hline Suppression period (s) & $1.4 \pm 0.1$ & $1.4 \pm 0.1$ & $1.4 \pm 0.0$ & $1.1 \pm 0.0$ \\
\hline \multicolumn{5}{|c|}{ Evoked activity after contralateral paw compression } \\
\hline Incidence (\%) & $50.0(8 / 16)$ & $54.5(6 / 11)$ & $54.5(6 / 11)$ & $45.5(5 / 11)$ \\
\hline Latency (ms) & $478.3 \pm 31.5$ & $419.3 \pm 33.8$ & $472.7 \pm 34.0$ & $402.2 \pm 63.9$ \\
\hline Spikes/s per burst & $3.6 \pm 0.3$ & $4.0 \pm 0.5$ & $3.5 \pm 0.2$ & $4.0 \pm 0.3$ \\
\hline Suppression period $(\mathrm{s})$ & $1.1 \pm 0.1$ & $1.3 \pm 0.2$ & $1.1 \pm 0.2$ & $1.3 \pm 0.1$ \\
\hline \multicolumn{5}{|l|}{ Effective dose 50} \\
\hline UK14,304 ( $\mu \mathrm{g} / \mathrm{kg})$ & $21.8 \pm 2.9[6]$ & $16.6 \pm 3.0[4]$ & $21.0 \pm 3.3[6]$ & $21.2 \pm 3.9[5]$ \\
\hline Reversion (\%) & $81.4 \pm 5.3[6]$ & $87.7 \pm 26.6[3]$ & $96.5 \pm 12.6[6]$ & $128.2 \pm 26.5[4]$ \\
\hline Desipramine ( $\mathrm{mg} / \mathrm{kg})$ & $0.3 \pm 0.1[7]$ & $0.2 \pm 0.0[5]$ & $0.3 \pm 0.1[7]$ & $0.2 \pm 0.0[8]$ \\
\hline Reversion (\%) & $33.7 \pm 5.0[4]$ & $35.1 \pm 8.2[4]$ & $40.6 \pm 11.4[7]$ & $40.8 \pm 4.7[7]$ \\
\hline
\end{tabular}

CCI, chronic constriction injury.

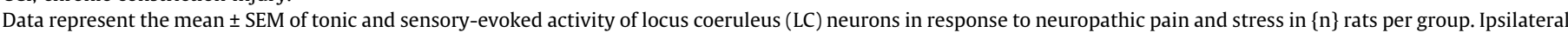

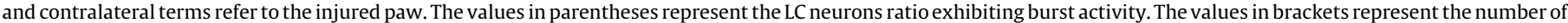

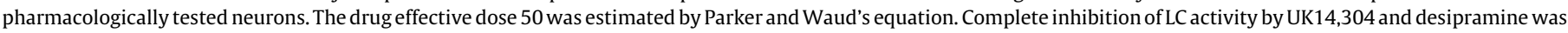

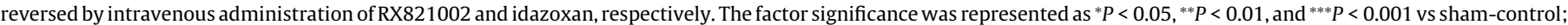
${ }^{+} P<0.05$ vs sham-stress, ${ }^{\#} P<0.05$ and ${ }^{\# \#} P<0.01$ vs CCI-control. The burst incidence was analyzed by Fisher's exact test: ${ }^{*} P<0.05$ vs sham-control.
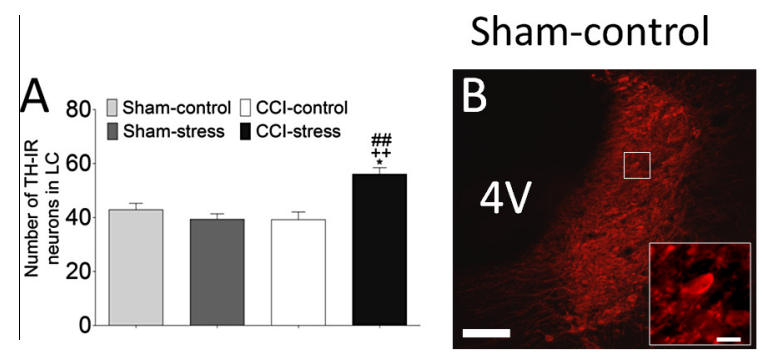

Sham-stress

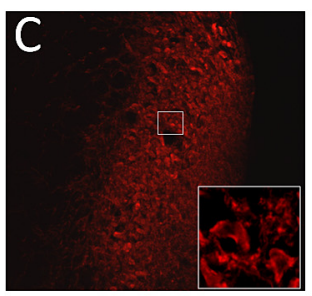

$\mathrm{F}$
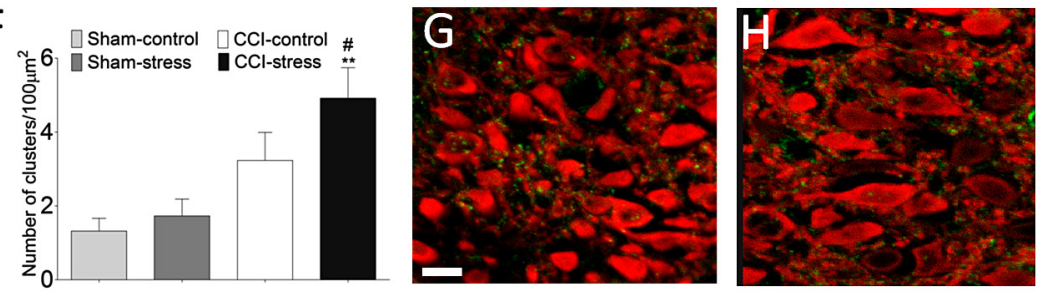

CCl-control
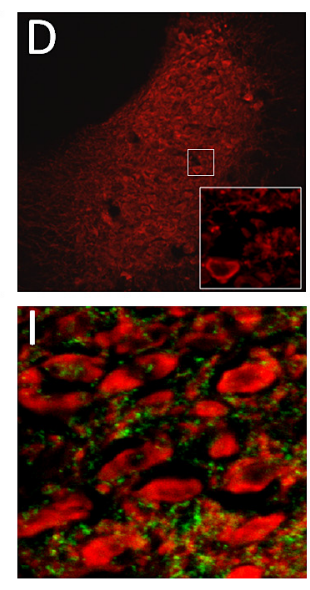

$\mathrm{CCl}$-stress
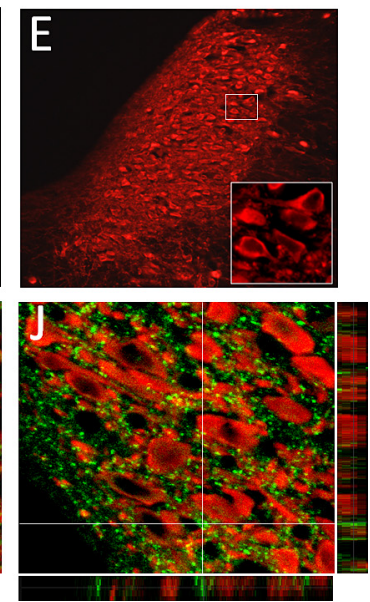

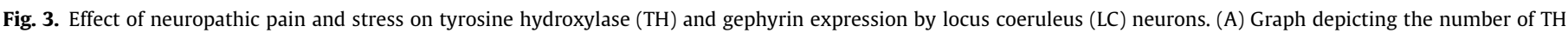

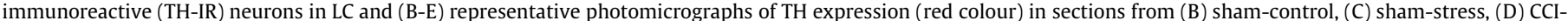

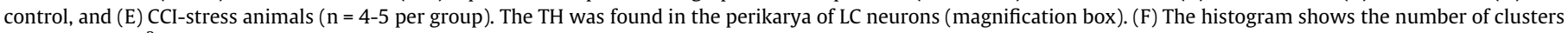

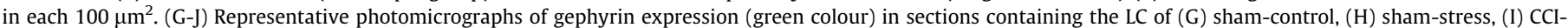

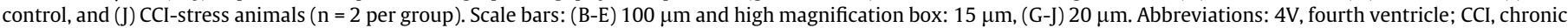
constriction injury. The data are expressed as the mean \pm SEM. ${ }^{*} P<0.05$ vs sham-control; ${ }^{++} P<0.01$ vs sham-stress; ${ }^{\#} P<0.05$ and ${ }^{\# \#} P<0.01$ vs CCI-control.

respectively). Nevertheless, anxiety-like behaviour was not modified in any group, because there were no differences in the time spent in the open zone in the elevated zero maze (Fig. 1E) and the number of close zone entries was similar in all groups, precluding a motor component in the observed effects (Fig. 1F). Similarly, no differences were found in the marble-burying test (Fig. 1G).

\subsection{Electrophysiological activity of LC neurons}

We evaluated any possible noradrenergic dysfunction by exploring the tonic (spontaneous) firing of contralateral LC neurons
(Table 2). Neither nerve injury (CCI-control) nor social isolation (sham-stress) modified LC firing activity. However, when pain and stress were combined (CCI-stress), they induced a lower tonic firing rate and burst activity than that displayed by sham-operated and CCI-controls $(P<0.001, P<0.05$, and $P<0.01$, respectively: Table 2 and Fig 2D), suggesting a decrease in activity of LC neurons. Furthermore, ISI histograms from sham-control, sham-stress, and CCI-control groups showed a similar Gaussian distribution of about $0.5 \mathrm{~ms}$ (Fig. 2F), reflecting a normal discharge pattern. However, the CCI-stress group presented an obvious rightward shift in this distribution with a greater number of ISI at intervals of about 1.0-1.5 ms (Fig. 2G). This abnormal distribution of ISI was 
corroborated by a reduction of the variation coefficient of CCIstress (Table 2).

Alpha2-adrenoceptors play an important role in controlling the firing activity of LC neurons, and consequently, in noradrenaline release from noradrenergic fibres. Indeed, i.v. administration of UK14,304 or desipramine inhibited spontaneous activity in a dose-dependent manner, producing a similar $\mathrm{ED}_{50}$ and dose-response curve in all experimental groups (Fig. 2H-I). Complete inhibition of LC neuron activity by UK14,304 and desipramine $\left(E_{\max }=100 \%\right)$ was reversed by i.v. administration of the $\alpha_{2}$-adrenoceptor antagonists, RX821002 (100\%) and idazoxan (30\%), respectively. Therefore, no differences were found regarding $\alpha_{2^{-}}$ adrenoceptors function among groups (Table 2).

When we evaluated the sensory-evoked (phasic) activity of the LC during ipsilateral paw compression, evoked discharge was slightly enhanced in CCI animals when measured through the number of spikes per burst. However, in CCI animals also subjected to stress, both spikes per burst and the incidence of the response augmented significantly (Fig. 2E). This effect was accompanied by a shortening of the latency (Table 2 and Fig. 2J-K). These differences were not found when the contralateral hind paw was compressed (Table 2).

\section{3. $T H$ and gephyrin expression at the LC Level}

In another group of animals, we explored TH expression in the LC by immunofluorescence, revealing that there was a significant upregulation in the expression of TH in the LC (bilaterally) of the CCI-stress animals with respect to the sham-control, sham-stress, and CCI-control $(P<0.05, P<0.01, P<0.01$ respectively: Fig. 3A, $B-E)$. By contrast, the animals that experienced just pain or stress did not show any changes in the number of TH-IR neurons when compared to the sham-control group. When we evaluated the expression of gephyrin, a multifunctional protein selectively located at inhibitory synapses [15] (Fig. 3F, G-J), there was also a significant increase expression in CCI-stress when compared to the sham-control or sham-stress rats $(P<0.01$ and $P<0.05$, respectively, Fig. $3 F$ ). The expression of gephyrin was restricted to the dendritic domains of TH-IR (Fig. 3J), which are specifically GABAergic inhibitory innervations [14].

\subsection{Effect of intra-LC bicuculline administration}

As gephyrin expression was augmented in CCI-stress animals and since the inhibitory modulation of LC neurons is thought to be mediated mainly through GABA-A receptors, we evaluated the effect of intra-LC (contralateral) bicuculline administration (Fig. 4D). Bicuculline significantly decreased the time spent by CCI-stressed animals in the white area (Fig. 4C), while it did not modify the time spent in the white area of any of the sham-operated animals (Fig. 4B). Additionally, this effect seemed to be specific because no motor or behavioural impairment was evident in this test (Speed in the white area $[\mathrm{cm} / \mathrm{s}]$ : Vehicle: Sham-control: $4.4 \pm 0.8$, Sham-stress: $7.3 \pm 1.6$, CCI-control: $5.9 \pm 1.1$, CCIstress: $4.3 \pm 0.7$; Bicuculline: Sham-control: $5.9 \pm 1.1$, Shamstress: $3.1 \pm 1.3$, CCI-control: $4.8 \pm 1.0$, CCI-stress: $4.1 \pm 0.4$ ). Moreover, intra-LC administration of bicuculline did not modify the mechanical nociceptive pain threshold in any group (Fig. 4A).

\section{Discussion}

Stress is considered a psychobiological factor that may have an important adverse influence on the course of chronic pain. The present study demonstrates that socially isolated neuropathic rats suffer worse affective pain and that this effect is modulated by LC neurons.

We have explored the effect of being socially isolated on the different dimensions of neuropathic pain perception. In the sensory-discriminative component, our results show that $\mathrm{CCI}$ rats developed the same level of allodynia irrespective of whether they were stressed by isolation. To evaluate the affective-cognitive elements, we have used the PEAT. This test evaluates the aversive nature of a noxious stimulus based on the avoidance of a preferred location where the stimulus is delivered. Thus, the animals have to choose between a dark nonanxiogenic area in which the injured paw is stimulated and the white mildly anxiogenic region in which the noninjured paw is stimulated [23]. As expected, we found that CCI-controls spend more time in the white area than the shamcontrol or sham-stress groups. However, the CCI-stress group spent even more time in the white area, with the maximum avoidance score. It is interesting to note that this effect was already evident within 5 minutes after starting the test, suggesting that the animals are highly focused on pain avoidance, and that stress increases the aversion to noxious stimulation.

It appears that anxiety did not compromise the results obtained in the PEAT, as all the animals tested showed similar levels of anxiety when evaluated in the elevated zero maze and marbleburying tests. We previously found that 2 weeks of neuropathic pain and chronic mild stress selectively increased the aversion to painful stimuli, without affecting classic depressive-like behaviours measured through the forced swimming and anhedonia test [10]. Thus, these and previous data corroborate that it takes approximately 4 weeks for rats to develop affective disorders (anxiety and depression) in association with neuropathic pain $[2,19,37,43]$. Nevertheless, further studies will be necessary to explore the effect of stress on the pain dimensions and secondary affective disorders over longer periods.

Regarding LC function, tonic LC discharge activity was not modified by either CCI or stress, consistent with previous data $[1,11,22]$. However, the basal firing rate and bursting activity highlighted a significant decrease in the spontaneous activity of LC neurons in CCI-stress. As the decrease of tonic activity could be mediated by indirect activation of inhibitory $\alpha_{2}$-adrenoceptors [25], we checked for possible overactivation of these receptors. However, the dose-response curves of the $\alpha_{2}$-adrenoceptor agonist UK14,304 and the noradrenaline reuptake inhibitor desipramine were similar in all groups, ruling out such a hypothesis. When we evaluated the expression of $\mathrm{TH}$, the rate-limiting enzyme of noradrenaline synthesis, there was no change after 5 weeks in isolation or 2 weeks of neuropathic pain, consistent with the electrophysiological data. An increase in TH has previously been seen in the LC after stressful stimuli [26]. However, in other studies no such changes were detected (using chronic footshock or restraint stress [36]). This might also be the case in our model of stress, possibly because this short period of isolation alone is insufficient to trigger a stress response in adult animals or alternatively, because adaptive changes had already developed. On the other hand, while we did not find any change in TH expression 2 weeks after CCI, an increase was evident in group-housed rats after 4 weeks [2]. This suggests that isolation might advance the changes observed 4 weeks after neuropathy is induced. However, it is important to note that although both conditions lead to an increase in $\mathrm{TH}$ expression, the electrophysiological situation is quite different. A decrease in tonic and burst firing was observed in CCI-stressed rats after 2 weeks, while there was no change in tonic activity, but bursting activity and $\alpha_{2}$-adrenoceptors sensitivity was enhanced in CCI-group-housed rats after 4 weeks [2]. Whereas behaviourally isolated rats did not show any change in anxiety, their pain experience appeared to be heightened. By contrast, after 4 weeks, CCI grouped rats consistently showed anxio-depressive-like behaviours [2] that were in accordance with the LC changes in the brain tissue of depressed patients seen postmortem [28,29,45]. Together, these data indicate that specific LC dysregulation might be linked to different behavioural outcomes. It will be interesting to 
determine if isolated CCI animals at 4 weeks will develop anxiodepressive disorders similar to those already described for CCIgrouped housed rats, and whether they are accompanied by comparable alterations in the LC. Such information will help to link behavioural alterations to specific brain signatures.

Neuropathic pain was previously shown to be associated with higher glutamic acid decarboxylase expression and GABA release in the LC [44]. Accordingly, gephyrin expression was slightly enhanced by nerve injury, but this expression was significantly robust when nerve injury was associated with stress, and it was accompanied by functional changes such as lower and irregular tonic discharge. In addition, when a noxious mechanical stimulus was applied to the injured paw, there was a trend in the CCI-control animals to increase the burst firing rate of the contralateral LC, consistent with our previous data using an electrical train stimulus [3]. As with the other parameters studied, this effect was very pronounced in the CCI-stress group. Interestingly, no differences among groups were found when the noninjured paw was stimulated, suggesting that the exaggerated evoked response is restricted to the nociceptive input from the injury (ascending pain pathway). Therefore, CCI-stress altered the pattern of LC discharge to noxious stimuli such that the ratio of evoked/tonic activity increased, especially when the injured paw is stimulated. We propose that this might effectively enhance the LC-forebrain signalling temporally linked to noxious events, increasing the attention to pain perception in CCI-stress. Indeed, studies in patients have shown that attention to pain enhances perceived pain $[34,42]$. It has been shown in awake animals that the LC facilitates different behavioural outcomes by shifting between tonic and phasic modes of discharge $[4,40]$. Thus, CCI-stress may shift the physiological response of the LC-noradrenergic system toward a phasic state when the injured paw is stimulated. This displacement would augment the activation of the LC by a noxious stimulus and consequently produce hyperarousal to perceived pain and stronger avoidance to re-experience pain in conditions of CCI-stress in the PEAT. That is, CCI-stress group has a more negative interpretation of painful experiences in spite of similar sensorial pain and anxiety threshold. Interestingly, it has been suggested in animal models that the enhanced ratio of evoked/tonic activity of LC neurons is causing the exaggerated response to trauma-related stimuli of posttraumatic stress disorder [18]. A similar pattern was been found in the LC of adult rats subjected to resident-intruder social stress model [9].

Although several neurotransmitters have been shown to modulate tonic and sensory-evoked LC activity (eg, excitatory amino acids, GABA, corticotropin-release-factor), our data as well as previous data point to an enhanced GABAergic neurotransmission in the LC in neuropathic pain $[38,44]$. Thus, we explored the effect
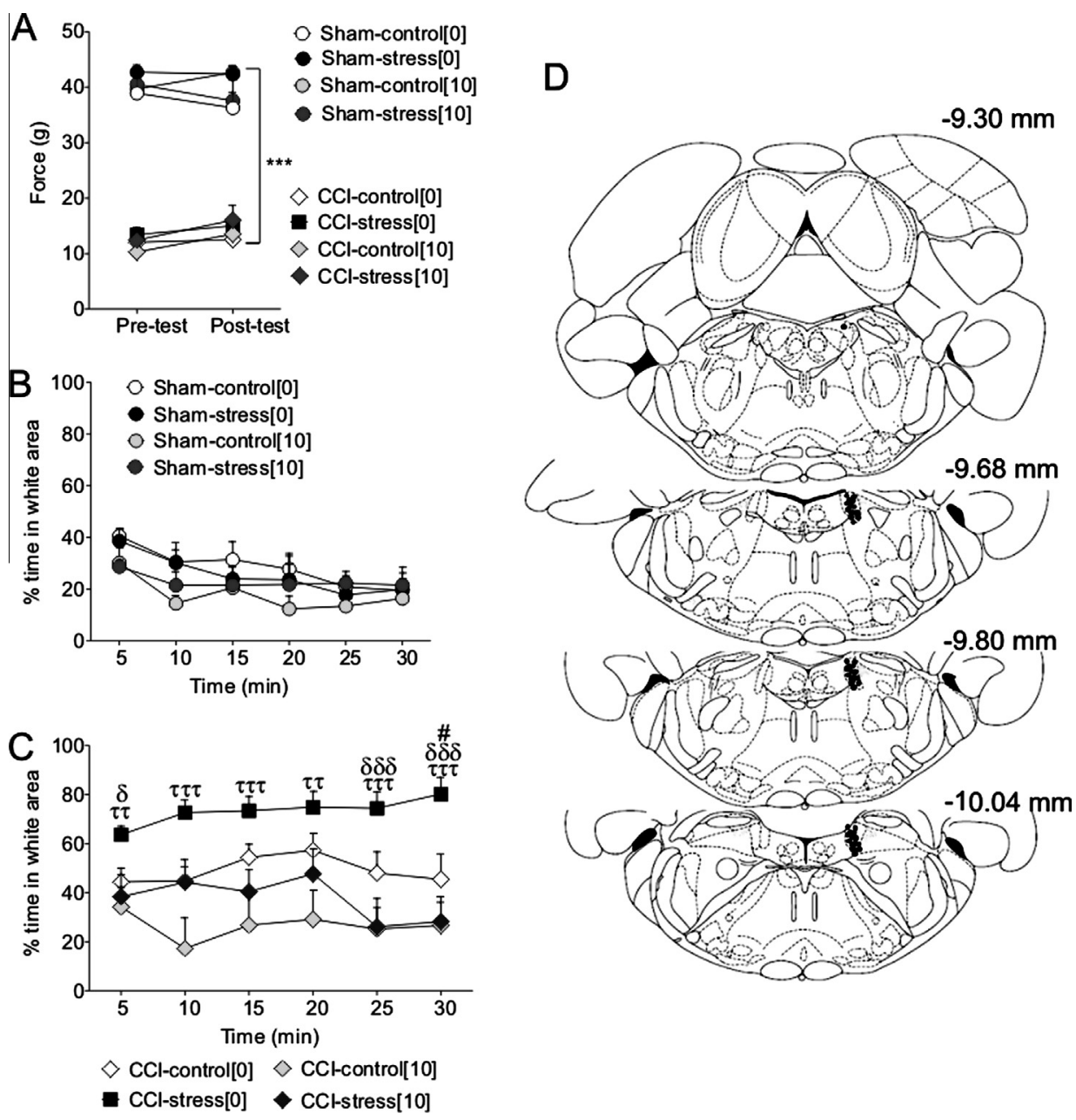

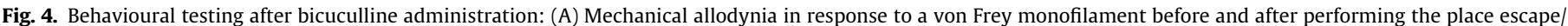

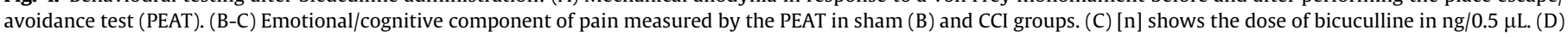

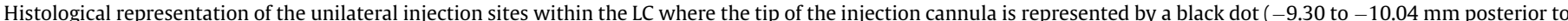

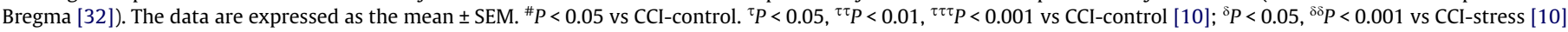
( $n=7-11$ per group). 
of GABA-A receptors blockade in the contralateral LC because GABA signals mainly through these receptors [14,30]. Results showed that the intra-LC administration of bicuculline, a GABA-A receptor antagonist, diminished the time spent in the white area in the PEAT. Altogether, this suggests that GABA blockade at LC level attenuated the negative affective effects of pain inputs, probably due to a decrease of evoked/tonic activity ratio in the LC. Further studies in the ipsilateral LC are necessary to make a definitive statement about the role of GABA neurotransmission in the ascending and descending pain pathway mediated by LC.

Current conceptualization of chronic pain is toward the integration of the obvious sensorial aspects, with other more diffuse, such as psychological and social, variables that may explain different patients' outcomes [13]. In the current study, we have used animals to model pain perception when social network is removed and could be translated to those neuropathic pain patients that have or perceive altered social support (lack of supportive relationships [hostility from family and/or friends], marital problems, lack of financial support, belonging to disadvantaged groups [homeless]). Our results show that when neuropathic pain is associated with social isolation stress, there is a modified LC reaction to noxious stimuli that exacerbate the response. As a consequence, this greater reactivity to pain stimulus compromises the ability of an individual to adapt to new contingencies. This might be an adaptive $\mathrm{LC}$ response that aims to enhance the negative pain experience as a defensive reaction. These data, jointly with previous ones about anxio-depressive-like disorders developed at long-term neuropathic pain, are placing the brain noradrenaline system as a key area mediating mood alterations in chronic pain.

\section{Conflict of interest}

The authors declare no conflict of interest.

\section{Acknowledgements}

We would like to thank Mrs Raquel Rey-Brea, Mrs Clara MuñozMediavilla, and Mr Jesus Gallego-Gamo for their excellent technical assistance. This study was supported by grants from the Fondo de Investigacion Sanitaria (PI10/01221, PI12/00915), CIBERSAM (G18), Junta de Andalucía-Consejería de Innovación, Ciencia y Empresa (CTS-510, CTS-4303 and CTS-7748), Cátedra Externa del Dolor Fundación Grünenthal-Universidad de Cádiz, FP7-PEOPLE2010-RG (268377), and FPU fellowship (AP2007-02397).

\section{References}

[1] Alba-Delgado C, Borges G, Sanchez-Blazquez P, Ortega JE, Horrillo I, Mico JA, Meana JJ, Neto F, Berrocoso E. The function of alpha-2-adrenoceptors in the rat locus coeruleus is preserved in the chronic constriction injury model of neuropathic pain. Psychopharmacology (Berl) 2012;221:53-65.

[2] Alba-Delgado C, Llorca-Torralba M, Horrillo I, Ortega JE, Mico JA, SanchezBlazquez P, Meana JJ, Berrocoso E. Chronic pain leads to concomitant noradrenergic impairment and mood disorders. Biol Psychiatry 2013;73: 54-62.

[3] Alba-Delgado C, Mico JA, Sanchez-Blazquez P, Berrocoso E. Analgesic antidepressants promote the responsiveness of locus coeruleus neurons to noxious stimulation: implications for neuropathic pain. PAIN ${ }^{\circledR} 2012 ; 153$ : 1438-49.

[4] Aston-Jones G, Cohen JD. An integrative theory of locus coeruleusnorepinephrine function: adaptive gain and optimal performance. Annu Rev Neurosci 2005;28:403-50.

[5] Bennett GJ, Xie YK. A peripheral mononeuropathy in rat that produces disorders of pain sensation like those seen in man. PAIN ${ }^{\circledR} 1988 ; 33: 87-107$.

[6] Berrocoso E, De Benito MD, Mico JA. Role of serotonin 5-HT1A and opioid receptors in the antiallodynic effect of tramadol in the chronic constriction injury model of neuropathic pain in rats. Psychopharmacology (Berl) 2007;193:97-105.

[7] Berrocoso E, Mico JA. In vivo effect of venlafaxine on locus coeruleus neurons: role of opioid, alpha(2)-adrenergic, and 5-hydroxytryptamine(1A) receptors. J Pharmacol Exp Ther 2007;322:101-7.
[8] Berrocoso E, Mico JA, Vitton O, Ladure P, Newman-Tancredi A, Depoortere R, Bardin L. Evaluation of milnacipran, in comparison with amitriptyline, on cold and mechanical allodynia in a rat model of neuropathic pain. Eur J Pharmacol 2011;655:46-51.

[9] Bingham B, McFadden K, Zhang X, Bhatnagar S, Beck S, Valentino R. Early adolescence as a critical window during which social stress distinctly alters behavior and brain norepinephrine activity. Neuropsychopharmacology 2011;36:896-909.

[10] Bravo L, Mico JA, Rey-Brea R, Perez-Nievas B, Leza JC, Berrocoso E. Depressivelike states heighten the aversion to painful stimuli in a rat model of comorbid chronic pain and depression. Anesthesiology 2012;117:613-25.

[11] Brightwell JJ, Taylor BK. Noradrenergic neurons in the locus coeruleus contribute to neuropathic pain. Neuroscience 2009;160:174-85.

[12] Cedarbaum JM, Aghajanian GK. Noradrenergic neurons of the locus coeruleus: inhibition by epinephrine and activation by the alpha-antagonist piperoxane. Brain Res 1976;112:413-9.

[13] Chapman CR, Tuckett RP, Song CW. Pain and stress in a systems perspective: reciprocal neural, endocrine, and immune interactions. J Pain 2008;9:122-45

[14] Corteen NL, Cole TM, Sarna A, Sieghart W, Swinny JD. Localization of GABA-A receptor alpha subunits on neurochemically distinct cell types in the rat locus coeruleus. Eur J Neurosci 2011;34:250-62.

[15] Essrich C, Lorez M, Benson JA, Fritschy JM, Luscher B. Postsynaptic clustering of major GABAA receptor subtypes requires the gamma 2 subunit and gephyrin. Nat Neurosci 1998;1:563-71.

[16] Flatters SJ, Bennett GJ. Ethosuximide reverses paclitaxel- and vincristineinduced painful peripheral neuropathy. PAIN ${ }^{\circledR} 2004 ; 109: 150-61$.

[17] Flor H, Hermann C. Biopsychosocial models of pain. In: Dworkin RH, Breitbart WS, editors. Psychosocial aspects of pain: a handbook for health care providers, vol. 27. Seattle: IASP Press; 2004. p. 47-76.

[18] George SA, Knox D, Curtis AL, Aldridge JW, Valentino RT, Liberzon I. Altered locus coeruleus-norepinephrine function following single prolonged stress. Eur J Neurosci 2013;37:901-9.

[19] Goncalves L, Silva R, Pinto-Ribeiro F, Pego JM, Bessa JM, Pertovaara A, Sousa N, Almeida A. Neuropathic pain is associated with depressive behaviour and induces neuroplasticity in the amygdala of the rat. Exp Neurol 2008;213: 48-56.

[20] Grace AA, Bunney BS. The control of firing pattern in nigral dopamine neurons: burst firing. J Neurosci 1984;4:2877-90.

[21] Grant MM, Weiss JM. Effects of chronic antidepressant drug administration and electroconvulsive shock on locus coeruleus electrophysiologic activity. Biol Psychiatry 2001;49:117-29.

[22] Jedema HP, Finlay JM, Sved AF, Grace AA. Chronic cold exposure potentiates CRH-evoked increases in electrophysiologic activity of locus coeruleus neurons. Biol Psychiatry 2001;49:351-9.

[23] LaBuda CJ, Fuchs PN. A behavioral test paradigm to measure the aversive quality of inflammatory and neuropathic pain in rats. Exp Neurol 2000;163: 490-4.

[24] Leonard BE. Stress, norepinephrine and depression. J Psychiatry Neurosci 2001;26:S11-6.

[25] Mateo Y, Meana JJ. Determination of the somatodendritic alpha2-adrenoceptor subtype located in rat locus coeruleus that modulates cortical noradrenaline release in vivo. Eur J Pharmacol 1999;379:53-7.

[26] Nestler EJ, Alreja M, Aghajanian GK. Molecular control of locus coeruleus neurotransmission. Biol Psychiatry 1999;46:1131-9.

[27] Njung'e K, Handley SL. Evaluation of marble-burying behavior as a model of anxiety. Pharmacol Biochem Behav 1991;38:63-7.

[28] Ordway GA, Schenk J, Stockmeier CA, May W, Klimek V. Elevated agonist binding to alpha2-adrenoceptors in the locus coeruleus in major depression. Biol Psychiatry 2003;53:315-23.

[29] Ordway GA, Smith KS, Haycock JW. Elevated tyrosine hydroxylase in the locus coeruleus of suicide victims. J Neurochem 1994;62:680-5.

[30] Osmanovic SS, Shefner SA. Gamma-aminobutyric acid responses in rat locus coeruleus neurones in vitro: a current-clamp and voltage-clamp study. J Physiol 1990;421:151-70.

[31] Parker RB, Waud DR. Pharmacological estimation of drug-receptor dissociation constants. Statistical evaluation: I. Agonists. J Pharmacol Exp Ther 1971;177: $1-12$.

[32] Paxinos G, Watson C. The rat brain in stereotaxic coordinates. 4th ed. London: Academic Press; 1998.

[33] Pertovaara A. Noradrenergic pain modulation. Prog Neurobiol 2006;80:53-83.

[34] Quevedo AS, Coghill RC. Attentional modulation of spatial integration of pain: evidence for dynamic spatial tuning. J Neurosci 2007;27:11635-40.

[35] Sassoe-Pognetto M, Follesa P, Panzanelli P, Perazzini AZ, Porcu P, Sogliano C, Cherchi $\mathrm{C}$, Concas A. Fluctuations in brain concentrations of neurosteroids are not associated to changes in gephyrin levels. Brain Res 2007;1169:1-8.

[36] Smith MA, Brady LS, Glowa J, Gold PW, Herkenham M. Effects of stress and adrenalectomy on tyrosine hydroxylase mRNA levels in the locus ceruleus by in situ hybridization. Brain Res 1991;544:26-32.

[37] Suzuki T, Amata M, Sakaue G, Nishimura S, Inoue T, Shibata M, Mashimo T. Experimental neuropathy in mice is associated with delayed behavioral changes related to anxiety and depression. Anesth Analg 2007;104:1570-7. table of contents.

[38] Takasu K, Ono H, Tanabe M. Gabapentin produces PKA-dependent presynaptic inhibition of GABAergic synaptic transmission in LC neurons following partial nerve injury in mice. J Neurochem 2008;105:933-42. 
[39] Turk DC, Audette J, Levy RM, Mackey SC, Stanos S. Assessment and treatment of psychosocial comorbidities in patients with neuropathic pain. Mayo Clin Proc 2010;85:S42-50

[40] Valentino RJ, Van Bockstaele E. Convergent regulation of locus coeruleus activity as an adaptive response to stress. Eur J Pharmacol 2008:583:194-203.

[41] West CH, Ritchie JC, Boss-Williams KA, Weiss JM. Antidepressant drugs with differing pharmacological actions decrease activity of locus coeruleus neurons. Int J Neuropsychopharmacol 2009;12:627-41.

[42] Wiech K, Ploner M, Tracey I. Neurocognitive aspects of pain perception. Trends Cogn Sci 2008;12:306-13.
[43] Yalcin I, Bohren Y, Waltisperger E, Sage-Ciocca D, Yin JC, Freund-Mercier MJ, Barrot M. A time-dependent history of mood disorders in a murine model of neuropathic pain. Biol Psychiatry 2011;70:946-53.

[44] Yoshizumi M, Parker RA, Eisenach JC, Hayashida K. Gabapentin inhibits gamma-amino butyric acid release in the locus coeruleus but not in the spinal dorsal horn after peripheral nerve injury in rats. Anesthesiology 2012;116:1347-53.

[45] Zhu MY, Klimek V, Dilley GE, Haycock JW, Stockmeier C, Overholser JC, Meltzer HY, Ordway GA. Elevated levels of tyrosine hydroxylase in the locus coeruleus in major depression. Biol Psychiatry 1999;46:1275-86. 